\title{
Cantinas de escolas estaduais de Curitiba/PR, Brasil: adequação à lei de regulamentação de oferta de alimentos
}

\author{
Canteens of State Schools in Curitiba in the State of Paraná, Brazil: \\ adaptation to the food supply regulation law
}

Christiane Opuszka Machado (http://orcid.org/0000-0002-1676-4342) ${ }^{1}$

Doroteia Aparecida Höfelmann (https://orcid.org/0000-0003-1046-3319) ${ }^{2}$

${ }^{1}$ Secretaria Municipal de Saúde. R. Francisco Torres 830, Centro. 80060-130 Curitiba PR Brasil.christianeopuszka@ gmail.com

${ }^{2}$ Programa de PósGraduação em Saúde Coletiva, Universidade Federal do Paraná. Curitiba PR Brasil.

\begin{abstract}
The scope of this study was to determine the food on offer in state public school canteens of Curitiba, Paraná, and to investigate the association between the permitted/prohibited food supplied under the Canteen Law, with school and canteen variables. A cross-sectional study was conducted with interviews with canteen administrators in 27 state schools. The Mann Whitney test was used to investigate associations. There was a higher frequency of administration of the canteens under the self-management regime ( $n=$ 25, 92.6\%); appropriate location in the school for serving meals ( $n=20,74.1 \%)$; length of time in administration of over 10 years $(n=13,48.2 \%)$ and administrators who reported knowing the Canteen Law $(n=22,81.5 \%)$. More than 2/3 of canteens sold prohibited food, such as sweetened beverages ( $n=22,81.5 \%)$ and candies, industrialized popcorn and salty snacks $(n=13,48.2 \%)$. Only one school provided fried snacks $(n=1$, $3.7 \%$ ). The offer of technical education alone was associated with greater availability of food allowed by the legislation ( $p=0.033$ ). The school canteens evaluated can be described as places of commercialization of food not permitted by the Healthy Canteen Law. It is important to highlight the regulation and inspection of food sales in canteens for the promotion of health in schools.

Key words Food services, School food, Enforcement of the law
\end{abstract}

Resumo Objetivou-se caracterizar a oferta de alimentos nas cantinas de escolas públicas estaduais de Curitiba, Paraná, e investigar associação da oferta de alimentos permitidos/não permitidos pela Lei das Cantinas, com variáveis da escola $e$ da cantina. Foi realizado estudo transversal com entrevista aos administradores de cantinas em 27 escolas estaduais e foi utilizado o teste de Mann Whitney para investigar associações. Verificou-se maior frequência de administração das cantinas por autogestão $(n=25 ; 92,6 \%)$; local adequado na escola para realização das refeições $(n=20$; 74,1\%); tempo na administração do local superior a 10 anos $(n=13 ; 48,2 \%)$ e de administradores que referiram conhecer a Lei $(n=22 ; 81,5 \%)$. Mais de 2/3 das cantinas comercializava alimentos não permitidos, tais como bebidas açucaradas ( $n=22 ; 81,5 \%)$ e doces, pipocas e salgadinhos industrializados ( $n=13 ; 48,2 \%)$. Apenas uma (3,7\%) ofertava salgados fritos. Somente a oferta de ensino técnico associou-se à maior disponibilidade de alimentos permitidos pela legislação ( $p=$ $0,033)$. As cantinas avaliadas podem ser caracterizadas como locais de oferta de alimentos não permitidos pela Lei da Cantina Saudável. Destaca-se a importância da regulação e da fiscalização da comercialização de alimentos nas cantinas para promoção de saúde nas escolas.

Palavras-chave Serviços de alimentação, Alimentação escolar, Aplicação da lei 


\section{Introdução}

Há mais de uma década, além de estudos internacionais ${ }^{1-4}$ e nacionais ${ }^{5,6}$, organismos internacionais, como a Organização Mundial de Saúde $(\mathrm{OMS})^{7}$, vem apresentando o ambiente escolar como um local estratégico de enfrentamento da obesidade e estímulo à prática de alimentação saudável.

Em 2015, no Brasil, a escola fazia parte da realidade de $97,7 \%$ das crianças e adolescentes entre 6 e 14 anos e de $84,3 \%$ dos adolescentes entre 15 e 17 anos, que frequentavam o Ensino Fundamental e Médio, respectivamente ${ }^{8}$. Este caráter universal permite que a escola se apresente como espaço privilegiado de convívio, troca de experiências e aprendizados, incluindo aqueles relacionados à educação nutricional e à alimentação escolar. Neste sentido, a escola deve se configurar tanto como promotora quanto inibidora de comportamentos alimentares que levem à obesidade ${ }^{9}$.

Independentemente de como e onde é adquirida, a alimentação escolar brasileira inclui todo e qualquer alimento ofertado neste ambiente durante o período letivo ${ }^{10}$. As cantinas, a alimentação gratuita ofertada por meio da execução do Programa Nacional de Alimentação Escolar (PNAE), os lanches adquiridos no entorno e aqueles provenientes de casa competem entre si, dentro deste espaço, pelas preferências alimentares dos alunos ${ }^{11}$.

Diante de um cenário de prevalência elevada de sobrepeso e obesidade entre os escolares brasileiros $^{12-14}$, a promoção da alimentação saudável aparece como prioridade das políticas públicas, entre elas a Política Nacional de Promoção à Saú$\mathrm{de}^{15}$, a Política Nacional de Alimentação e Nutri$c_{\tilde{a}} \mathrm{o}^{16} \mathrm{e}$ a Portaria Interministerial no $1.010^{10}$.

Ao analisar as políticas públicas de nutrição brasileiras com potencial de contribuir na intervenção da obesidade infantil, um amplo estudo de revisão, com publicações realizadas entre 1990 e 2010 sobre o tema, apontou o ambiente escolar como local promissor para ações eficazes de promoção e prevenção da obesidade e a regulamentação de venda e propaganda de alimentos nesse ambiente, como uma das principais medidas de efeito $^{6}$. Os alimentos e as bebidas que não são ofertados gratuitamente nas escolas são considerados por alguns autores como "alimentos competitivos", uma vez que podem interferir no consumo da alimentação saudável ofertada por meio do PNAE ${ }^{11,17,18}$. Um dos estudos, realizado com alunos de dez municípios brasileiros de grande e médio porte, observou, inclusive, que a existên- cia de cantinas comerciais nas escolas era inversamente proporcional à adesão diária ao $\mathrm{PNAE}^{19}$.

Por esta razão, alguns estados e municípios implementaram políticas próprias de regulamentação do comércio de alimentos nas cantinas ${ }^{20}$. $\mathrm{O}$ estado do Paraná instituiu a Lei no 14.423 , de 02 de junho de $2004^{21}$, e a Lei no $14.855^{22}$, de 19 de outubro de 2005, que regulamentam a presença destes estabelecimentos e o tipo de alimentos comercializados por eles nas escolas públicas e particulares.

As leis do estado do Paraná proibiram a comercialização de diversos produtos nas cantinas escolares, entre eles: bebidas com quaisquer teores alcoólicos; balas, pirulitos e gomas de mascar; chocolates; refrigerantes e sucos artificiais; salgadinhos industrializados e biscoitos recheados; salgados fritos (coxinha, rissoles, croquete) e pipocas industrializadas, além de obrigar estes estabelecimentos comerciais a ofertar diariamente ao menos dois tipos de frutas ${ }^{21,22}$.

Conhecer o perfil destas cantinas pode propiciar informações importantes para subsidiar atividades de intervenção, destinadas a melhorar a qualidade dos alimentos ofertados no ambiente escolar e, com isto, atuar sobre o consumo da alimentação escolar gratuita, promoção de hábitos saudáveis e da prevenção do excesso de peso. Além disto, também permite avaliar se a Lei da Cantina Saudável ${ }^{22}$, por meio da limitação da oferta de alimentos não saudáveis e estímulo à oferta de alimentos saudáveis, cumpre, após uma década de implementação, sua função de promoção à saúde.

Portanto, o objetivo deste artigo foi caracterizar a oferta de alimentos nas cantinas de escolas públicas estaduais de Curitiba, Paraná, e investigar associação da oferta de alimentos permitidos e não permitidos pela Lei das Cantinas, com variáveis da escola e da cantina.

\section{Métodos}

Estudo transversal de base escolar, parte do projeto "Excesso de peso e características do ambiente escolar em estudantes de Curitiba/PR", conduzido in loco entre os meses de março de 2016 e abril de 2017, nas cantinas de escolas públicas estaduais do município de Curitiba, capital do Paraná.

As estimativas de tamanho de amostra para o projeto maior consideraram: população estimada de 110.238 estudantes na faixa etária de 10 a 19 anos, matriculados na rede pública estadual em Curitiba, nos turnos diurnos, prevalência 
desconhecida de desfecho de 50\%, margem de erro de 4 pontos percentuais, nível de confiança de $95 \%$ e efeito de delineamento amostral de 2 , totalizando 1.194 estudantes. Considerando-se possíveis perdas ou recusas, a esse valor foi acrescido $20 \%$ e o tamanho amostral final passou a ser de 1.433 estudantes.

A partir do tamanho da amostra optou-se por realizar amostragem com o mesmo tamanho para cada escola $(n=48)$, o que resultou em seleção aleatória de 30 escolas, das 162 existentes em lista disponibilizada pela Secretaria da Educação do Paraná ${ }^{23}$. As escolas de educação especial ou indígena foram excluídas do estudo. Neste artigo são apresentados dados das cantinas escolares inseridas nas instituições de ensino sorteadas.

Os dados foram obtidos por meio de entrevista estruturada com os administradores das cantinas escolares e aplicação de instrumento validado de auditoria de estabelecimentos de comercialização de alimentos para consumo imediato (Healthy Restaurant Index - HMRI), desenvolvido por Duran et al. ${ }^{24}$. Ao instrumento foram acrescidas questões para avaliação da execução da Lei no 14.855/05, a Lei da Cantina Saudável do Estado do Paraná ${ }^{2}$. Previamente à coleta de dados foi realizado teste piloto em uma cantina não sorteada para avaliar a necessidade de ajustes no instrumento e/ou procedimentos de coleta de dados. Apenas o instrumento foi modificado, com a supressão de uma questão que se encontrava em duplicata e a inclusão de uma questão sobre o alimento mais vendido pela cantina.

Foram utilizadas no instrumento questões fechadas e abertas para observar os seguintes aspectos: 1) ambiente do estabelecimento; 2) cardápio; 3$)$ preço dos produtos; 4) propagandas; e 5) informações sobre o funcionamento das cantinas.

Os dados do ambiente do estabelecimento foram classificados de modo dicotômico (não, sim) em relação a presença de: opções de pratos quentes e frios no cardápio; buffet de hortaliças cruas; informação nutricional sobre os produtos oferecidos em murais ou folders; mural com informações sobre alimentação saudável e; existência de local apropriado na escola para a realização das refeições.

Sobre o cardápio foram verificados aspectos dicotômicos (não, sim) relativos à: presença exclusiva de serviço de rodízio ou self-service; existência de informação nutricional das preparações e; oferta de salada como prato principal, acompanhamento ou como pratos exclusivos. Quando existentes, foram também observadas dicotomicamente (não, sim): possibilidade de substituir batata frita por hortaliças sem custo adicional; opção de pedir porções menores por valor reduzido; presença de frutas in natura ou saladas de frutas no cardápio; presença de sucos naturais de frutas e possibilidade de substituir refrigerante por suco natural sem custo adicional. Neste item também foi provida uma listagem com os alimentos proibidos $(\mathrm{n}=12)$ e permitidos $(n=21)$ pela Lei das Cantinas ${ }^{22}$, bem como foi verificado o número de opções de salgados assados, salgados fritos e de sanduíches naturais disponíveis.

O preço de batatas fritas, salgados fritos, salgados assados, refrigerantes e frutas foi investigado pelos pesquisadores. Também foi avaliado se existia igualdade nos valores cobrados entre o suco natural e o refrigerante.

Nos locais, os pesquisadores observaram a existência de propagandas sobre sucos, frutas e/ ou hortaliças ou sobre refrigerantes, batata frita, sobremesas e/ou sorvetes.

Entre as informações da cantina, os administradores foram questionados sobre conhecimento da Lei da Cantina Saudável (não, sim); modo de gestão do estabelecimento (Associação de Pais, Mestres e Funcionários e Similares (pessoa jurídica de direito privado, terceirizada, outras)); e tempo de administração da cantina (anos e meses). Questões abertas sobre quais alimentos consideravam saudáveis, dificuldades na oferta destes alimentos no estabelecimento, bem como na oferta de frutas e hortaliças, foram inseridas no instrumento. Adicionalmente, os administradores foram questionados em relação aos alimentos mais vendidos no estabelecimento.

Para a análise e avaliação dos produtos comercializados nas cantinas comerciais foi elaborado um sistema de pontuação para a classificação desses alimentos em permitidos ou não pela Legislação ${ }^{22}$. A classificação considerou as recomendações utilizadas na Lei da Cantina Saudável do Estado do Paraná ${ }^{2}$, que divide esses alimentos em dois grupos: permitidos e não permitidos, que podem ser associados, pela sua descrição, aos alimentos considerados saudáveis ou não-saudáveis. Alguns alimentos da listagem original foram agrupados, com o intuito de evitar sobreposição nas análises. $\mathrm{Na}$ listagem de alimentos permitidos, àqueles que apareceram apenas como ingredientes de sanduíches naturais foram agrupados no item sanduíches naturais com pão integral. $\mathrm{Na}$ listagem de alimentos não permitidos, foram agrupados os itens: alimentos com mais de $160 \mathrm{mg}$ de sódio em 100kcal do produto; alimentos com mais de $3 \mathrm{~g}$ de gordura em $100 \mathrm{kcal}$ do 
produto e; alimentos que contenham corantes e antioxidantes artificiais, visto que apareciam sempre que algum produto industrializado era comercializado.

Os alimentos permitidos e não permitidos pela Lei foram agrupados em dois índices distintos, gerados a partir da soma da listagem agrupada de alimentos permitidos $(\mathrm{n}=14)$ e não permitidos $(n=7)$ comercializados nas cantinas escolares, respectivamente. Ambos os índices obtiveram consistência interna, medida pelo Alpha de Cronbach, de 0,62.

Para a caracterização das escolas, o diretor ou responsável respondeu questionário estruturado, padronizado, anônimo e auto preenchível, modificado a partir do instrumento utilizado na PeN$\mathrm{SE}^{25}$. A partir dele foram obtidas informações sobre as características gerais da escola (número de alunos matriculados e níveis de ensino), sua estrutura física (presença de horta) e suas políticas escolares (existência de conselho escolar).

Após o preenchimento dos instrumentos os dados foram duplamente digitados em planilha eletrônica. Inicialmente foi utilizada análise descritiva dos dados (frequência, intervalo de confiança, média, desvio-padrão, mediana, valor máximo e valor mínimo). As correlações entre os índices de alimentos permitidos e não permitidos nas cantinas e demais variáveis contínuas foram investigadas por meio da correlação de Spearman. Associações entre os índices e variáveis categóricas foram investigadas por meio do teste de Mann Whitney. O nível de significância de $5 \%$ foi considerado nas análises, realizadas por meio do programa STATA, versão 12.0.

A pesquisa foi aprovada pelo Comitê de Ética em Pesquisa em Seres Humanos do Setor de Ciências da Saúde (CEP/SD) da Universidade Federal do Paraná e os participantes assinaram Termo de Consentimento Livre e Esclarecido, conforme as normas da resolução 466/12 do Conselho Nacional de Saúde.

\section{Resultados}

De um total de 30 escolas avaliadas, 3 (10,0\%) não apresentavam cantina escolar em funcionamento no dia da coleta dos dados, segundo relato dos diretores ou responsáveis. Não houve recusa por parte dos participantes.

Em sua maioria, as cantinas eram de responsabilidade da escola, administradas em regime de autogestão, por meio de representantes da Associação de Pais, Mestres e Funcionários (APMF)
( $\mathrm{n}=25 ; 92,6 \%)$; apresentavam local adequado na escola para a realização das refeições $(\mathrm{n}=20$; $74,1 \%)$ e seus administradores referiram conhecer a Lei da Cantina Saudável $(\mathrm{n}=22 ; 81,5 \%)$. O tempo na administração desses estabelecimentos variou de 1 mês a 71 anos (DP 15,5), contudo a maior parte dos locais estava sob a mesma administração há mais de 10 anos $(\mathrm{n}=13 ; 48,2 \%)$ (Tabela 1).

Aproximadamente $2 / 3$ das cantinas vendia alimentos não permitidos, com presença de corantes, alto teor de sódio (mais de $160 \mathrm{mg}$ em $100 \mathrm{kcal}$ do produto) e gordura (mais de $3 \mathrm{~g} \mathrm{em}$ $100 \mathrm{kcal}$ do produto). Refrigerantes, sucos artificiais e/ou refrescos $(n=22 ; 81,5 \%)$ foram encontrados na maior parte dos locais avaliados. Chocolates, gomas, caramelos, salgadinhos industrializados, biscoitos recheados e/ou pipocas industrializadas foram encontrados em praticamente metade das cantinas avaliadas. Apenas uma cantina comercializava salgados fritos. Em nenhuma cantina foi encontrado produto sem rotulagem ou sem data de validade (Tabela 2 ).

Dos itens permitidos, os mais encontrados foram os salgados assados $(n=25 ; 92,6 \%)$, seguido de bebidas lácteas $(n=18 ; 66,7 \%)$ e pães

Tabela 1. Características das cantinas de escolas estaduais de Curitiba, Paraná, 2016-2017.

\begin{tabular}{lc}
\hline \multicolumn{1}{c}{ Variável } & $\mathbf{n}(\%)$ \\
\hline $\begin{array}{l}\text { Tipo de gestão administrativa } \\
(\mathrm{n}=27)\end{array}$ & \\
$\quad$ Autogestão & $25(92,6)$ \\
$\quad$ Terceirizada & $2(7,4)$ \\
Tempo de Administração (anos) & \\
$(\mathrm{n}=27)$ & \\
$\quad$ Menos de 1 ano & $4(14,8)$ \\
& \\
$1-4,9$ anos & $7(25,9)$ \\
5 a 9,9 anos & $3(11,1)$ \\
$\quad$ Superior a 10 anos & $13(48,2)$ \\
Local adequado na escola para & \\
refeição (n = 27) & \\
Sim & $20(74,1)$ \\
Não & $7(25,9)$ \\
Conhece a Lei da Cantina Saudável & \\
(n=27) & \\
$\quad$ Sim & $22(81,5)$ \\
$\quad$ Não & $5(18,5)$ \\
Venda de salgado assado & \\
Número de variedades (n = 27) & $4,11(\mathrm{DP} 2,49)$ \\
Preço (R $\$)$ (n = 25) & $2,92(\mathrm{DP} 0,45)$ \\
\hline
\end{tabular}

Legenda: DP: desvio-padrão.

Fonte: as autoras (2017). 
Tabela 2. Alimentos permitidos e não permitidos comercializados nas cantinas de escolas estaduais de Curitiba, Paraná, 2016-2017. $(\mathrm{n}=27)$.

\begin{tabular}{|c|c|c|}
\hline Alimentos/Produtos & $\mathbf{n}$ & $\%$ \\
\hline \multicolumn{3}{|l|}{ Alimentos Permitidos } \\
\hline $\begin{array}{l}\text { Salgadinhos assados com pouco } \\
\text { teor de gordura }\end{array}$ & 25 & 92,6 \\
\hline $\begin{array}{l}\text { Bebidas lácteas, leite fermentado, } \\
\text { achocolatados }\end{array}$ & 18 & 66,7 \\
\hline $\begin{array}{l}\text { Pães em geral, pão de batata, pão } \\
\text { de queijo, pão doce recheado com } \\
\text { frutas ou geleia }\end{array}$ & 16 & 59,3 \\
\hline Picolé de frutas & 11 & 40,7 \\
\hline Chá mate, café & 9 & 33,3 \\
\hline $\begin{array}{l}\text { Bolacha "Maria"; biscoito de } \\
\text { maisena; "cream cracker", água e } \\
\text { sal, de polvilho, biscoito doce sem } \\
\text { recheio }\end{array}$ & 8 & 29,6 \\
\hline $\begin{array}{l}\text { Cereais integrais em flocos ou em } \\
\text { barras }\end{array}$ & 8 & 29,6 \\
\hline $\begin{array}{l}\text { Sanduíches naturais com pão } \\
\text { integral }\end{array}$ & 4 & 14,8 \\
\hline $\begin{array}{l}\text { Bolos de massa simples com recheio } \\
\text { de frutas, geleias e legumes }\end{array}$ & 3 & 11,1 \\
\hline Suco de frutas naturais & 2 & 7,4 \\
\hline Iogurte & 2 & 7,4 \\
\hline Água de coco & 2 & 7,4 \\
\hline \multicolumn{3}{|l|}{ Alimentos Não Permitidos } \\
\hline $\begin{array}{l}\text { Refrigerantes, sucos artificiais, } \\
\text { refrescos à base de pó } \\
\text { industrializado }\end{array}$ & 22 & 81,5 \\
\hline $\begin{array}{l}\text { Chocolates, doces à base de goma, } \\
\text { caramelos }\end{array}$ & 13 & 48,2 \\
\hline $\begin{array}{l}\text { Salgadinhos industrializados, } \\
\text { biscoitos recheados }\end{array}$ & 13 & 48,2 \\
\hline Pipocas industrializadas & 12 & 44,4 \\
\hline Balas, pirulitos e gomas de mascar & 6 & 22,2 \\
\hline Salgados folhados (tipo croissant) & 3 & 11,1 \\
\hline Salgados e doces fritos & 1 & 3,7 \\
\hline
\end{tabular}

Fonte: as autoras (2017).

( $\mathrm{n}=16 ; 59,3 \%$ ). Nenhuma cantina vendia os seguintes alimentos permitidos: frutas in natura ou secas, pasta de soja, atum/ovo cozido/requeijão, manteiga/margarina, creme vegetal e/ou chá mate/café. Queijo branco/ricota, frango/peito de peru e legumes/verduras foram encontrados como ingredientes dos sanduíches naturais com pão integral em quatro estabelecimentos, embora em um dos estabelecimentos avaliados sua comercialização estivesse restrita a professores e funcionários da escola (Tabela 2). Frutas in natura/saladas de frutas e sucos naturais de frutas foram encontrados em apenas uma escola. Duas escolas não vendiam salgados assados: uma vendia salgado frito e outra apenas pão de queijo. A média de número de opções de salgados assados foi de $4,11(\mathrm{DP} \pm 2,49)$, vendidos ao preço médio de $R$ 2,92 (DP $\pm 0,45)$.

Nenhuma cantina dispunha de buffet para oferta de preparações quentes (arroz, feijão, etc.) ou frias (saladas, frutas, sobremesas, etc.). Também não foi observada a presença de cartazes informativos/murais/folders sobre a qualidade dos alimentos e alimentação saudável. Apenas uma escola apresentava informação nutricional sobre os produtos oferecidos, mas a mesma não estava ainda disponível aos alunos, enquanto outra apresentava política de incentivo ao consumo de alimentos à base de verduras e legumes, por meio de oferta gratuita de pequenas porções para degustação.

Não foram observadas associações entre a presença de local adequado na escola para as refeições nas cantinas e os índices de alimentos permitidos e não-permitidos comercializados nos estabelecimentos. Também não foi encontrada associação destes índices com o conhecimento da Lei da Cantina Saudável pelo administrador, e tampouco com a presença de horta nas escolas. Apenas a oferta de ensino técnico esteve associada a maior disponibilidade de alimentos permitidos $(\mathrm{p}=0,033)$ (Tabela 3$)$.

Observou-se que a disponibilidade de alimentos permitidos nas cantinas esteve positivamente correlacionada ao número de alimentos não permitidos $(\mathrm{p}=0,017)$, ao número de alunos das escolas $(\mathrm{p}=0,189)$ e à duração do intervalo escolar $(p=0,048)$. Em relação à disponibilidade de alimentos não permitidos, foi verificada correlação positiva com a duração do intervalo escolar $(\mathrm{p}=0,231)$ e negativa com o número de opções de salgados $(\mathrm{p}=0,182)$ (Tabela 4$)$.

Frutas $(\mathrm{n}=15 ; 55,6 \%)$, sucos de frutas $(\mathrm{n}=$ $11 ; 40,7 \%)$ e salgados assados $(\mathrm{n}=7 ; 25,9 \%)$ foram apontados pelos entrevistados como opções de alimentos saudáveis a serem comercializados nas cantinas (dados não apresentados em tabelas).

Entre as razões apresentadas pelos administradores das cantinas para não ofertar frutas, verduras e legumes, prevaleceu a dificuldade de preparo destes alimentos $(\mathrm{n}=12 ; 44,4 \%)$, recusa de consumo pelos alunos $(n=8 ; 29,6 \%)$ e a oferta deles na alimentação escolar fornecida gratuitamente $(\mathrm{n}=4 ; 14,8 \%)$. Dificuldade de venda do produto ( $\mathrm{n}=3 ; 11,1 \%)$, de conservação do alimento $(\mathrm{n}=2 ; 7,4 \%)$ e falta de hábito por parte 
Tabela 3. Distribuição dos índices de alimentos permitidos e não permitidos de acordo com características das cantinas das escolas estaduais de Curitiba, Paraná, 2016-2017.

\begin{tabular}{|c|c|c|c|c|c|c|}
\hline \multirow{2}{*}{ Variáveis } & \multirow{2}{*}{$\mathbf{n}$} & \multicolumn{2}{|c|}{$\begin{array}{l}\text { Alimentos } \\
\text { Permitidos }\end{array}$} & \multirow{2}{*}{$\mathbf{n}$} & \multicolumn{2}{|c|}{$\begin{array}{c}\text { Alimentos Não } \\
\text { permitidos }\end{array}$} \\
\hline & & $\begin{array}{l}\text { Mediana } \\
(\mathrm{p} 25-\mathrm{p} 75)\end{array}$ & $\begin{array}{l}\text { Valor } \\
\text { de } p^{\star}\end{array}$ & & $\begin{array}{l}\text { Mediana } \\
(\mathrm{p} 25-\mathrm{p} 75)\end{array}$ & $\begin{array}{l}\text { Valor } \\
\text { de } p^{*}\end{array}$ \\
\hline \multicolumn{7}{|l|}{ Cantina } \\
\hline Conhece a Lei da Cantina Saudável $(\mathrm{n}=27)$ & & & 0,975 & & & 0,506 \\
\hline Não & 5 & $4,0(3,0-5,0)$ & & 5 & $5,0(4,0-6,0)$ & \\
\hline $\operatorname{Sim}$ & 22 & $4,0(3,0-5,0)$ & & 22 & $5,5(4,0-7,0)$ & \\
\hline Local adequado na escola para refeições $(n=27)$ & & & 0,443 & & & 0,097 \\
\hline Não & 7 & $4,0(3,0-4,0)$ & & 7 & $7,0(5,0-8,0)$ & \\
\hline Sim & 20 & $4,0(2,5-5,0)$ & & 20 & $5,0(4,0-6,0)$ & \\
\hline \multicolumn{7}{|l|}{ Escola } \\
\hline Tipo de gestão administrativa $(\mathrm{n}=27)$ & & & 0,420 & & & 0,573 \\
\hline Autogestão & 25 & $4,0(3,0-5,0)$ & & 25 & $5,0(4,0-7,0)$ & \\
\hline Terceirizada & 2 & $4,5(4,0-5,0)$ & & 2 & $6,0(6,0-6,0)$ & \\
\hline Oferta de ensino técnico $(\mathrm{n}=27)$ & & & $0,033^{*}$ & & & 0,674 \\
\hline Não & 20 & $4,0(2,5-4,0)$ & & 20 & $5,0(4,0-7,0)$ & \\
\hline $\operatorname{Sim}$ & 7 & $5,0(4,0-8,0)$ & & 7 & $6,0(4,0-7,0)$ & \\
\hline Presença de horta $(n=26)$ & & & 0,490 & & & 0,370 \\
\hline Não & 15 & $4,0(2,0-5,0)$ & & 15 & $5,0(4,0-7,0)$ & \\
\hline Sim & 11 & $4,0(3,0-5,0)$ & & 11 & $6,0(5,0-7,0)$ & \\
\hline
\end{tabular}

Legenda: ${ }^{\star}$ Testes de Mann Whitney.

Fonte: as autoras (2017).

Tabela 4. Correlação de Spearman entre os índices de alimentos permitidos e não permitidos e as variáveis contínuas das escolas e das cantinas estaduais de Curitiba, Paraná, 2016-2017.

\begin{tabular}{lcc}
\hline \multicolumn{1}{c}{ Variáveis } & $\begin{array}{c}\text { Alimentos } \\
\text { Permitidos }\end{array}$ & $\begin{array}{c}\text { Alimentos Não } \\
\text { permitidos }\end{array}$ \\
\hline $\begin{array}{l}\text { Alimentos } \\
\text { Permitidos } \\
\text { Alimentos Não- } \\
\text { permitidos }\end{array}$ & 1,00 & \\
Número Alunos & $0,43^{*}$ & 1,00 \\
$\begin{array}{l}\text { Duração do } \\
\text { intervalo escolar }\end{array}$ & $\mathbf{0 , 3 8}{ }^{\star}$ & $-0,01$ \\
$\begin{array}{l}\text { Tempo de } \\
\text { Administração da } \\
\text { cantina }\end{array}$ & $-0,05$ & 0,23 \\
$\begin{array}{l}\text { Número de } \\
\text { opções de } \\
\text { salgados assados }\end{array}$ & 0,11 & 0,04 \\
\hline $\begin{array}{l}\text { Legenda: }{ }^{*} \text { p }<0,05 . \\
\text { Fonte: as autoras }(2017) .\end{array}$ & \\
\end{tabular}

dos alunos $(\mathrm{n}=2 ; 7,4 \%)$, também foram citados. Apenas um administrador referiu não existir nenhuma dificuldade para a oferta desses alimentos (dados não apresentados em tabelas).

\section{Discussão}

Os resultados deste estudo mostraram que as cantinas de escolas estaduais de Curitiba podem ser consideradas locais de comercialização de lanches rápidos. Resultados semelhantes foram encontrados em estudos realizados em Porto Alegre $^{26}$, em Santa Catarina ${ }^{27}$ e no Distrito Federal $^{28}$. Nelas predomina o regime de autogestão com tempo de administração superior a 10 anos, resultado que diferiu dos estudos citados, que apontam uma prevalência de cantinas com gestão terceirizada. Esta diferença possivelmente se deve ao fato dos estudos avaliarem estabelecimentos tanto de escolas públicas, como de escolas privadas. Contudo, a predominância da autogestão não resultou em menor oferta de alimentos não permitidos pela legislação.

Apesar da Lei da Cantina Saudável ${ }^{22}$ estar em vigor, há mais de uma década, e ser de conhecimento da maior parte dos administradores, as cantinas comercializavam alimentos ultraprocessados em abundância e, em sua maioria, não ofereciam opções à base de frutas, verduras e legumes aos alunos. A dificuldade no preparo, a crença na recusa destas opções por parte dos alunos e o receio de que estes alimentos não fos- 
sem comercializados diante da oferta gratuita na alimentação do PNAE foram apontadas como razões para não comercializar alimentos considerados por eles como saudáveis.

A ausência, em quase sua totalidade, da venda de salgados fritos e de refrigerantes, destaca-se como fator positivo ${ }^{29}$. Outro aspecto positivo encontrado foi a inexistência de alimentos sem data de validade ou sem rotulagem. Porém, a presença maciça de comercialização de salgados assados, item permitido pela legislação ${ }^{22}$, deve ser analisada com parcimônia. Estes alimentos não foram avaliados em relação à sua composição nutricional e, conforme sua forma de processamento e da presença de alguns ingredientes em sua composição (farinha refinada, gordura hidrogenada, recheios à base de embutidos, queijos e molhos gordurosos), podem favorecer um padrão alimentar não saudável, caracterizado pela expressiva presença de gorduras trans, conservantes químicos e outras substâncias.

Por outro lado, era esperada uma maior frequência de oferta de frutas, visto a obrigatoriedade legal de oferta de duas opções diárias. A baixa oferta de frutas pelas cantinas foi encontrada em outros dois estudos ${ }^{29,30}$. Neste estudo, entre as cantinas das escolas avaliadas apenas uma apresentava política de incentivo ao consumo de alimentos à base de verduras e legumes, que consistia na distribuição gratuita de pequenas porções do produto. Estudo conduzido com jovens universitários ${ }^{31}$ mostrou que esta conduta aumenta significativamente o consumo de frutas e verduras.

Para além da disponibilidade dos alimentos, a OMS aponta o ambiente alimentar escolar como um local de promoção de hábitos saudáveis ${ }^{7}$. Alguns autores destacam que esse ambiente tem grande impacto nas escolhas alimentares dos adolescentes, de modo que a disponibilidade de alimentos e bebidas em ambientes escolares afeta a qualidade da dieta, a ingestão energética e o risco de obesidade ${ }^{11,32,33}$.

A complexidade de avaliar o ambiente alimentar escolar pode ser intensificada pelo compartilhamento do mesmo espaço para realização das refeições que envolvem a alimentação gratuita, e os produtos adquiridos na cantina comercial da escola, ou provenientes de outros locais. Além disto, observou-se correlação positiva entre a oferta de alimentos permitidos e não permitidos nas escolas avaliadas.

É importante destacar que as cantinas escolares, mesmo quando administradas pela APMF, são fonte de recursos financeiros para as escolas, os quais são aplicados na aquisição de materiais de consumo ou para realização de benfeitorias estruturais. Oferecer um cardápio de cantina escolar sustentável e saudável implica em pensar na qualidade da alimentação do aluno e não exclusivamente no lucro, o que requer o envolvimento da comunidade escolar ${ }^{28}$.

Incluídos nessa comunidade, os administradores da cantina necessitam de apoio para que a proposta de uma cantina mais saudável seja implementada e sustentável ao longo do tempo. Outros autores ${ }^{9,34}$ apontaram em seus estudos que as cantinas devem ser utilizadas como locais de educação alimentar e nutricional. Um deles ainda observou que ações de regulação para impedir a exposição da coletividade a fatores estimulantes de práticas não saudáveis são imprescindíveis ${ }^{9}$. Contudo, não se deve descartar a influência do comércio do entorno nas práticas alimentares não saudáveis ${ }^{35}$, visto que à medida que se controla o comércio da cantina, aumenta o comércio de alimentos não saudáveis nas proximidades das escolas.

As possibilidades de generalização deste estudo são restritas, pois o cálculo do tamanho da amostra foi delineado para ser representativo do número de alunos, e não do número de cantinas. A avaliação da disponibilidade de oferta ao invés da aquisição do produto em si ou do número de consumidores em cada estabelecimento é outra limitação a ser considerada. Estas limitações podem ter contribuído para que apenas a oferta de ensino técnico estivesse associada à maior disponibilidade de alimentos permitidos. Porém, ele apresenta contribuições no processo de sistematização das informações para caracterização de cantinas escolares.

Em conclusão, passados mais de 10 anos da implantação da Lei da Cantina Saudável nas escolas do Paraná, as cantinas das escolas estaduais avaliadas, independentemente do tipo de gestão, permanecem ofertando grande quantidade de alimentos com baixo teor de nutrientes e de alta densidade energética. Em contrapartida, ainda é tímida a participação de frutas, verduras e legumes no cardápio das cantinas. Considerando a alimentação como necessidade básica à manutenção da vida, é preciso discutir as estratégias de incentivo à oferta de alimentos saudáveis e de fiscalização. A limitação do acesso aos alimentos ultraprocessados é apenas uma das possibilidades de promoção de saúde nas escolas, de fortalecimento do direito humano à alimentação adequada e de controle do aumento da obesidade na infância e juventude. Outras possibilidades 
envolvem a Educação Alimentar e Nutricional, a escuta dos atores envolvidos na construção de um ambiente alimentar saudável e o estímulo à autonomia dos alunos na escolha por alimentos saudáveis.

\section{Colaboradores}

CO Machado e DA Höfelmann participaram diretamente no planejamento, execução e análises desse estudo. CO Machado e DA Höfelmann leram e aprovaram a versão final enviada. 


\section{Referências}

1. Williams CM, Nathan N, Delaney T, Yoong SL, Wiggers J, Preece S, Lubans N, Sutherland R, Pinfold J, Smith K, Small T, Reilly KL, Butler P, Wyse RJ, Wolfenden L. CAFÉ: a multicomponent audit and feedback intervention to improve implementation of healthy food policy in primary school canteens: protocol of a randomised controlled trial. BMJ Open [serial on the Internet]. 2015 Jun [cited 2017 Jun 01];5(6):[about 7 p.]. Available from: http://bmjopen.bmj.com/content/5/6/e006969.short

2. Mensink F, Schwinghammer SA, Smeets, A. The Healthy School Canteen Programme: a promising intervention to make the school food environment healthier. J Environ Public Health [serial on the Internet]. 2012 Mar [cited 2017 May 30];2012:[about 8 p.]. Available from: http://dx.doi. org/10.1155/2012/415746

3. Glickman D, Parker L, Sim LJ, Del Valle Cook H, Miller EA, editors. Committee on accelerating progress in obesity prevention: solving the weight of the Nation. Washington: National Academies Press; 2012.

4. Swinburn B, Egger G, Raza F. Dissecting obesogenic environments: the development and application of a framework for identifying and prioritizing environmental interventions for obesity. Prev Med [serial on the Internet]. 1999 [cited 2017 Jun 05];29(6):563570. Available from: http://dx.doi.org/10.1006/pmed. 1999.0585

5. Correa EN, Schmitz BAS, Vasconcelos FAG. Aspects of the built environment associated with obesity in children and adolescents: a narrative review. Rev Nutr 2015;28(3):327-340.

6. Reis CEG, Vasconcelos IAL, Barros JFN. Políticas públicas de nutrição para o controle da obesidade infantil. Rev Paul Pediatr [periódico na Internet]. 2011 [acessado 2017 Jun 05];29(4):625-633. Disponível em: http://www.scielo.br/pdf/rpp/v29n4/24.pdf

7. World Health Organization (WHO). Report of the commission on ending childhood obesity. Genebra: WHO; 2016.

8. Cruz P, Monteiro L, organizadores. Anuário Brasileiro da Educação Básica 2017. São Paulo: Ed. Moderna; 2017.

9. Schmitz BAS, Recine EGI, Cardoso GT, Silva JRM, Amorim NFA, Bernardon R, Rodrigues MLCF. A escola promovendo hábitos alimentares saudáveis: uma proposta metodológica de capacitação para educadores e donos de cantina escolar. Cad Saude Publica [periódico na Internet]. 2008 [acessado 2016 Jun 08];24(2):s312-s322. Disponível em: http://dx.doi. org/10.1590/S0102-311X2008001400016

10. Brasil. Portaria interministerial $\mathrm{n}^{\circ} 1.010$, de 8 de maio de 2006. Institui as diretrizes para a promoção da alimentação saudável nas escolas de educação infantil, fundamental e nível médio das redes públicas e privadas, em âmbito nacional. Diário Oficial da União 2006; 08 mai.

11. Story M, Nanney MS, Schwartz MB. Schools and obesity preventions: creating school environments and policies to promote healthy eating and physical activity. Milbank $Q$ [serial on the Internet]. 2009 [cited 2017 Feb 07];87:71-100. Available from: http://doi. org/10.1111/j.1468-0009.2009.00548.x
12. Brasil. Ministério da Saúde (MS). Vigitel Brasil, 2016: Vigilância de Fatores de Risco e Proteção para Doenças Crônicas por Inquérito Telefônico. Brasília: MS; 2017.

13. Instituto Brasileiro de Geografia e Estatística (IBGE). PeNSE: pesquisa nacional de saúde do escolar 2015. Rio de Janeiro: IBGE; 2016.

14. Costa LCF, Vasconcelos FAG, Corso ACT. Fatores associados ao consumo adequado de frutas e hortaliças em escolares de Santa Catarina, Brasil. Cad Saude Publica [periódico na Internet]. 2012 [acessado 2017 Mai 10];28:1133-1142. Disponível em: http://dx.doi. org/10.1590/S0102-311X2012000600012

15. Brasil. Ministério da Saúde (MS). Política Nacional de Promoção da Saúde (PNPS): revisão da Portaria MS/ $G M n^{\circ}$ 687, de 30 de março de 2006. Brasília: MS; 2015.

16. Brasil. Ministério da Saúde (MS). Política Nacional de Alimentação e Nutrição. Brasília: MS; 2012.

17. Leme ACB, Philippi ST, Toassa EC. O que os adolescentes preferem: os alimentos da escola ou os alimentos competitivos? Saude soc [periódico na Internet]. 2013 Jun [acessado 2017 Jun 09];22(2):456-467. Disponível em: http://dx.doi.org/10.1590/S010412902013000200016

18. Rosenheck, R. Fast food consumption and increased caloric intake: a systematic review of a trajectory towards weight gain and obesity risk. Obes Rev [serial on the Internet]. 2008 [cited 2017 Apr 04];9(6):535547. Available from: https://doi.org/10.1111/j.1467789X.2008.00477.x

19. Sturion GL, Silva MV, Ometto AMH, Furtuoso MCO, Pipitone MAP. Fatores condicionantes da adesão dos alunos ao Programa de Alimentação Escolar no Brasil. Rev Nutri [periódico na Internet]. 2005 [acessado 2016 Dez 14];18(2):167-181. Disponível em: http:// dx.doi.org/10.1590/S1415-52732005000200001

20. Brasil. Ministério da Saúde (MS). Experiências estaduais e municipais de regulamentação da comercialização de alimentos em escolas no Brasil: identificação e sistematização do processo de construção e dispositivos legais adotados. Brasília: MS; 2007.

21. Paraná. Lei no 14.423 , de 06 de junho de 2004. Dispõe que os serviços de lanches nas unidades educacionais públicas e privadas que atendam a educação básica, localizadas no Estado, deverão obedecer a padrões de qualidade nutricional e de vida, indispensáveis à saúde dos alunos. Diário Oficial do Paraná, 2004; 06 jun.

22. Paraná. Lei no 14.855 , de 19 de outubro de 2005 Dispõe sobre padrões técnicos de qualidade nutricional, a serem seguidos pelas lanchonetes e similares, instaladas nas escolas de ensino fundamental e médio, particulares e da rede pública. Diário Oficial do Paraná, 2005; 19 out.

23. Paraná. Secretaria da Educação do Paraná (SEP). Superintendência de Desenvolvimento Educacional. Diretoria de Políticas e Tecnologias Educacionais. Consulta Escolas - 2014. Paraná: SEP; 2015.

24. Duran ACFL, Latorre M, Jaime, PC. Development of a Healthy Meal-Restaurant Index and a Healthy Retai Food Store Index: Obesogenic Environment Brazilian Study (ESAO). AAEA/EAAE Food Environment Symposium. Boston, MA, Agricultural and Applied Economics Association. 2012, May 30-31;123389. 
25. Instituto Brasileiro de Geografia e Estatística (IBGE). Pesquisa Nacional de Saúde do Escolar - 2012. Rio de Janeiro: IBGE; 2013.

26. Willhelm FF, Ruiz E, Oliveira ABA. Cantina Escolar: qualidade nutricional e adequação à legislação vigente. Clinical \& Biomedical Research [periódico na Internet]. 2010 Out [acessado 2010 Jun 10];30(3). Disponível em: http://seer.ufrgs.br/index.php/hcpa/ article/view/15375

27. Gabriel CG, Santos MV, Vasconcelos FAG, Milanez GHG, Hulse SB. Cantinas escolares de Florianópolis: existência e produtos comercializados após a instituição da Lei de Regulamentação. Rev Nutr [periódico na Internet]. 2010 [acessado 2015 Ago 12];23:191199. Disponível em: http://dx.doi.org/10.1590/S141552732010000200002

28. Porto EBS, Schmitz BAS, Recine EGI, Rodrigues MLCF. School canteens in the Federal District, Brazil and the promotion of healthy eating. Rev Nutr [serial on the Internet]. $2015 \mathrm{Fev}$ [cited 2017 Jan 13];28(1):29-41. Available from: http://dx.doi. org/10.1590/1415-52732015000100003

29. Gabriel CG, Vasconcelos FA, Andrade DF, Schmitz BAS. First Law regulating school canteens in Brazil: evaluation after seven years of implementation. Archi Latinoam Nutr [serial on the Internet]. 2009 [cited 2016 Nov 27];59(2):128-138. Available from: https:// www.ncbi.nlm.nih.gov/pubmed/19719008

30. Gaetani RS, Ribeiro LC. Produtos comercializados em cantinas escolares do município de Ribeirão Preto. Revista Brasileira em Promoção da Saúde 2015; 28(4):587-595.

31. Lachat CK, Verstraeten R, De Meulenaer B, Menten J, Huybregts LF, Van Camp J, Roberfroid D, Kolsteren PW. Availability of free fruits and vegetables at canteen lunch improves lunch and daily nutritional profiles: a randomized controlled trial. $\mathrm{Br} J \mathrm{Nutr}$ [serial on the Internet]. 2009 Oct [cited $2016 \mathrm{Nov}$ 08];102(7):1030-1037. Available from: http://dx.doi. org/10.1017/S000711450930389X
32. Briefel RR, Crepinsek MK, Cabili C, Wilson A, Gleason PM. School food environments and practices affect dietary behaviors of U.S. public school children. $J$ Am Diet Assoc [serial on the Internet]. 2009 [cited 2015 Sep 09];109(2):91-107. Available from: https:// www.ncbi.nlm.nih.gov/pubmed/19166677

33. Templeton SB, Marlette MA, Panemangalore M. Competitive foods increase the intake of energy and decrease the intake of certain nutrients by adolescents consuming school lunch. J Am Diet Assoc [serial on the Internet]. 2005 Jan [cited $2016 \mathrm{Jul} 28$ ];105(2):215220. Available from: http://dx.doi.org/10.1016/j. jada.2004.11.027

34. Roset MA, Gonzalvo B. School meals in Catalonia: surveillance and quality control. Public Health Nutr [serial on the Internet]. 2001 Dec [cited 2017 Jan 21];4(6a):1339-1341. Available from: https://www. ncbi.nlm.nih.gov/pubmed/11918476

35. Amorim NFA, Schmitz BAS, Rodrigues MLCF, Recine EGI, Gabriel CG. Implantação da cantina escolar saudável em escolas do Distrito Federal, Brasil. Rev Nutr [periódico na Internet]. 2012 Mar-Abr [acessado 2017 Mar 23];25:203-217. Disponível em: http:// dx.doi.org/10.1590/S1415-52732012000200003

Artigo apresentado em 13/06/2017

Aprovado em 05/03/2018

Versão final apresentada em 07/03/2018 